\title{
Pluralismo, estructuración y construcción de la identidad en la educación media uruguaya: interacciones desde las trincheras
}

Adriana Marrero*

\section{Resumen}

El problema de la interacción entre adultos y jóvenes dentro de las instituciones educativas, sobre todo en los niveles medios, ha sido un objeto de estudio usual por parte de psicólogos y sociólogos alrededor del mundo. Uno de los más relevantes e influyentes trabajos en este campo es Aprendiendo a trabajar, de Paul Willis, el que ha sido seguido por un número importante de estudios similares. El artículo toma la aproximación de Willis como punto de partida de una discusión que toma también en consideración dos de los principales conceptos de la Sociología actual, como es el de estructura y acción (Giddens) y, más recientemente, la idea de Margaret Archer sobre la "conversación interna". Desde allí, el artículo analiza las formas en las cuales los estudiantes de educación media uruguayos se relacionan con los adultos en el contexto del debilitamiento de las reglas específicamente educativas dentro de las escuelas. Desde el punto de vista metodológico, el artículo se basa en varias series de entrevistas a docentes y estudiantes de educación media, y en la observación directa de la vida y de las relaciones sociales dentro de los liceos montevideanos durante el período 1991-2003.

Palabras clave: Educación Media; Interacción entre jóvenes y adultos; Uruguay.

\section{Pluralism, structuring, and construction of identity in Uruguayan secondary education: interactions from the trenches}

\section{Abstract}

The problem of interaction between adults and youngsters inside educational institutions, mostly in secondary education, has been a frequent object of study by Psychologists and Sociologists around the world. One of the most relevant and influential productions in this field is Paul Willis' Learning to Labor, which was followed by an important number of studies of the same kind. The article takes Willis' approach as point of departure of a discussion that takes also into consideration two of the main concepts of today's Sociology, namely the ideas of structure and action (Giddens) and, more recently, Margaret Archer's idea of the "internal conversation". From that point of view, the article analyses the forms in which Uruguayan high school students interact with adults in the context of the weakening of educational rules inside schools. From a methodological perspective, the article is based on a series of interviews with high school teachers and students, and on the direct observation of the life and social relations in Montevideo's high schools in the period of 1991-2003.

Keywords: Secondary Education; Youngsters-adults interaction; Uruguay.

* Professora Doutora da Universidad de la República, Montevideo, Uruguay. 


\section{Adriana Marrero}

\section{Introducción}

En un artículo reciente, uno de los más lúcidos intérpretes de la cultura juvenil en las instituciones escolares - Paul Willis - denominaba a los jóvenes "los soldados rasos de la modernidad", "involuntarios y desorientados conscriptos en batallas que nunca son explicadas" (WILLIS, 2007, p. 109). En la primera línea de fuego de unos conflictos iniciados desde otros campos-económicos, políticos, tecnológicos - los jóvenes responden como pueden, pero dentro de unas pautas que no carecen de lógica ni de pertinencia, a las posibilidades reales que les presenta un mundo sobre el que tienen escaso o nulo control. En este espacio escolar, escenario privilegiado de esas otras luchas, es donde se expresa también la sobrevivencia, la mortandad o la simple deserción de los jóvenes, en la turbia e incierta carrera hacia el logro laboral y social.

La metáfora bélica, que aplicada al campo educativo puede en un principio resultar tan asombrosa como chocante, nos alerta, sin embargo, sobre las múltiples tensiones y líneas de fractura que se acumulan en el espacio de la escuela, y sobre los modos cómo los sufren y sobreviven las personas concreas que lo habitan. Mucho más acá de las notables apreciaciones de Willis - que ligan la experiencia educativa con las transformaciones globales y locales de las diversas olas de la modernidad- la metáfora nos sirve además, para visualizar las luchas casi siempre invisibles que se originan en el propio ámbito escolar, y los modos cómo se dirimen y procesan estos conflictos.

Además, la idea de "trinchera", que he tomado como título, tiene dentro de la perspectiva teórica adoptada acá, una utilidad adicional: la de incorporar - por la vía de la referencia a una actitud de lucha - la subjetividad de los propios actores, la que usualmente queda teóricamente relegada, y frecuentemente olvidada, frente a los potentes pero descarnados conceptos clásicos de estructura y acción.

Veremos estas cuestiones tanto desde un punto de vista teórico, como empírico. Para ello, partiremos de la presentación de cinco situaciones problemáticas que pueden considerarse como casos típicos de lo que es la vida cotidiana de la educación media en Uruguay, que servirán de referencia al punto de partida conceptual y al análisis final. Conceptualmente, partiremos de: a) la conformación de la identidad en el contexto del pluralismo de sentido en la segunda modernización, y b) una noción de la estructura social, tal como es desarrollada por A. Giddens y M. Archer. Desde allí, intentaremos arrojar luz a los casos presentados, mediante el análisis de los modos de articulación y desencuentro entre las formas específicamente escolares de estructuración del espacio social, y las formas plurales y múltiples que no le son específicas, y que afloran a través de distintos actores y en diferentes momentos. 
Pluralismo, estructuración y construcción de la identidad en la educación media uruguaya: interacciones desde las trincheras

\section{El nivel de la experiencia: docentes y alumnos que hablan y actúan}

- El 7 de mayo de 2008, en el Liceo No. 38 de La Teja, un grupo de estudiantes tiró petardos y encendió una papelera. Desde la Dirección del Liceo, se optó por un abordaje punitivo y general: dejar a todos los jóvenes encerrados, sin recreo. Frustrados, los estudiantes protestan. La respuesta de la Dirección, se sale del cauce institucional: se llama a la fuerza policial para que actúe. Los docentes se desresponsabilizan a sí mismos: culpan a los jóvenes y a sus familias; las autoridades del Consejo de Secundaria no creen - en lo inmediato- que deban hacer acto de presencia. Los docentes suspenden las clases, no por un día, sino por dos, no para los revoltosos - de una clase- sino para todo el liceo.

- Se estima que un tercio de las clases de educación media en institutos públicos se pierden por la inasistencia de los docentes, y es bien sabido que a este nivel, los profesores no suelen encargar a sus estudiantes más trabajos que los reglamentariamente establecidos: un trabajo escrito por bimestre - tres o cuatro a lo largo del año, y aún así, muchas veces no hacen una devolución adecuada de los mismos a sus estudiantes. Un estudiante de educación media, dice: "Quisiera que los docentes se tomaran con más seriedad su asistencia y que se hagan cargo de sus horas desde el comienzo de los cursos. Que se respete a los alumnos como nosotros a los profesores" (MARRERO, 2008, p. 66)

- En alianza tácita con los malos estudiantes, una profesora de práctica - durante mi formación como docente- aprovecha el desorden que se genera en el salón cuando llega tarde, para usar el resto del tiempo de clase para "rezongar" a los supuestos revoltosos, que resultan siendo todos. La docente elige no dar una clase que probablemente no tuviera preparada; los malos estudiantes se salvan de ser interrogados sobre una lección que no han estudiado; los buenos estudiantes son los que pierden.

- En la mayoría de los liceos públicos, las paredes de las aulas, pero también los corredores, y por supuesto, los baños, están repletas de "pintadas" de todo tipo, tamaño y color; el comportamiento de los estudiantes dentro de los salones, condice, con frecuencia, con el decorado de las paredes. Como dice una docente: "Andá a (tal liceo) hace 13 años que estoy... allá no puede aprender nadie así tengas a Einstein parado ahí., yo no te puedo explicar lo que eran los salones de clase... Todo lo que tú podés imaginarte estaba escrito en las paredes, lo que hacían los alumnos a nadie le importaba, que esos alumnos gritaran, escupieran, eructaran, todo eso mientras transcurría el examen de didáctica" (MARRERO, 2008, p. 170). 


\title{
Adriana Marrero
}

¿Qué nos dicen estos casos sobre la vida de estudiantes y de docentes dentro de la institución educativa uruguaya, a nivel medio? ¿Cómo puede leerse con un común marco de interpretación, estos fenómenos, aparentemente diversos, sometidos lógicas y pautas contradictorias? Esperamos dar respuesta a estas cuestiones en los próximos apartados.

\section{El marco conceptual: pluralismo, crisis de sentido y estructuración... una vez más}

\section{a) El contexto cultural: Modernidad, pluralismo y crisis de sentido}

No por ser un diagnóstico ya asentado, deja de ser pertinente recordar que la principal consecuencia de la modernización cultural, fue el pluralismo de sentido, esto es, la pérdida de marcos valorativos comunes sobre los que se construyen las identidades singulares y en relación con los cuales adquieren significado los cursos de acción concretos, y los proyectos vitales de los sujetos. En la primera modernidad, el pluralismo fue visto como un residuo disperso de la secularización, y como resultado del avance racionalizador que se inicia, o bien por la incorporación de las creencias religiosas a las prácticas cotidianas y su posterior olvido (como en Weber) o por la expansión de la lógica competitiva de la explotación capitalista (como en Marx), pero siempre como expresión de un proceso de vastos alcances y de profundas consecuencias subjetivas, por el cual "todo lo sólido se desvanece en el aire".

\begin{abstract}
El pluralismo moderno conduce a la relativización total de los sistemas de valores y esquemas de interpretación. Dicho de otro modo: los antiguos sistemas de valores y esquemas de interpretación se han "descanonizado" [...] el pluralismo es la causa de la crisis de sentido en la modernidad. (BERGER; LUCKMANN, 1997, p. 75-76)
\end{abstract}

El ascenso y multiplicación de cultos del más diverso tipo, y en otras latitudes, la exacerbación de las lógicas recíprocas de los radicalismos religiosos, han relegado a un discreto segundo plano a la idea misma de secularización. Pero ello no ha mermado la presencia del pluralismo en nuestras sociedades. Radicalizado o no, en sus formas puras, sincréticas, o de simple pastiche, incluso lo religioso ha venido a constituirse en un "mercado" diverso y caótico donde todo puede adquirirse y combinarse en distintas proporciones y por variados períodos de tiempo. Si todo vale, nada vale de verdad, y el sujeto, desorientado, se ve obligado a construir, por sí, puntos de referencia que den sentido a su experiencia y a su futuro.

En este contexto, las identidades ya no se conforman por la interiorización de normas a través de canales tradicionales de socialización. La erosión de la tradición, la atención al cambio, la búsqueda de adecuación y la adaptabilidad constantes, la convivencia con personas diferentes y el contacto con costumbres distintas, el acceso por los mass media a una enorme variedad 
de formas de vida y de pensamiento, en fin, las distintas formas del pluralismo que socavan el conocimiento que se daba por supuesto, causan desorientación y desosiego. La necesidad de orientación provee de nuevas funciones a las principales instituciones sociales - la familia, la escuela - encargadas ahora, además, de la producción y mediación de sentido social.

Esto tiene una singular pertinencia en el campo educativo, al que se le adjudica un papel central en la constitución y trasmisión de un sistema de valores y normas que es constitutivo del orden social y jurídico al que pertenece. La escuela, formadora de futuros adultos autónomos, generadora de ciudadanía, capacitadora de trabajadores, tiene como cometido central contribuir a la conformación de identidades singulares, pero sobre todo colectivas, siempre provisorias, pero dotadas de instrumentos para la propia orientación en mundos complejos y cambiantes. Los jóvenes, sometidos a estímulos múltiples y contradictorios provenientes de distintas fuentes, desorientados e inseguros, necesitan, claro está, de estas instituciones productoras y mediadoras de sentido social legitimado - de la escuela más que de ninguna otra - para la construcción de una identidad que no dependa sólo del vaivén más o menos espasmódico de las múltiples influencias a las que está sometida su experiencia en un contexto cultural de sobre-estimulación sensorial, pluralismo y relativismo valorativo.

Pero ¿qué ocurre cuando esas instituciones educativas están pobladas de adultos igualmente desorientados, confundidos e inseguros? Porque finalmente, ¿por qué el pluralismo, el relativismo y la crisis de sentido, así como los múltiples y contradictorios mensajes de los medios, habrían de afectar sólo a los jóvenes, y no también a los adultos de su misma sociedad?

Este es el contexto de unos conflictos que son difícilmente comprensibles fuera de él. Bien mirados, las luchas que se entablan en el sistema educativo, pocas veces tienen que ver con el autoritarismo de los docentes, o con el arcaísmo de un sistema de valores que se pretende imponer sin más, sino más bien, con unos espacios educativos vacilantes, fluctuantes, casi holográficos, donde casi todo es posible porque pocas cosas son reales, con un mundo de apariencias insustancial, donde los educadores no confían en sus propias capacidades o conocimientos, y a veces, ni siquiera sobre su derecho a educar, porque todo lo que de sólido tenía la institución educativa, parece haberse desvanecido, ya, en el aire. Volveremos sobre esto más adelante.

\section{b) Los aspectos relevantes: estructura, acción y el sujeto que conversa consigo mismo}

Pensar el campo educativo desde la sociología, requiere de su percepción como espacio estructurado. Para ello, nos interesan dos aspectos de la teoría de la estructuración de Giddens, la que requiere ser complementada con la idea de "conversación interna" de Archer. 
a) La dualidad de la estructura: Para Giddens, la estructura consiste en las "propiedades por las que se vuelve posible que prácticas sociales discerniblemente similares existan a lo largo de segmentos variables de tiempo y de espacio, y que presten a estos una forma sistémica" (GIDDENS, 1995a, p. 54), o en "conjuntos de reglas-recursos que intervienen en el ordenamiento institucional de sistemas sociales" (GIDDENS,1995a, p. 396). Así vista, la estructura sólo existe en y por medio de las actividades de los actores: "en tanto huellas mnémicas y en tanto ejemplificada en prácticas sociales, es en cierto aspecto más 'interna' que exterior, en un sentido durkheimiano a las actividades de ellos. Estructura no se debe asimilar a constreñimiento sino que es a la vez constrictiva y habilitante" (GIDDENS, 1995a, p. 61). Esto significa que no es posible separar conceptual o empíricamente la acción de la estructura, ya que: "el momento de producción de la acción es también un momento de reproducción en los contextos donde se escenifica cotidianamente la vida social. [...] La estructura no existe con independencia del saber que los agentes poseen sobre lo que hacen en su actividad cotidiana" (GIDDENS,1995a, p. 396). A esta doble característica de la estructura, la de constituir y ser constituida, le llama Giddens "la dualidad de la estructura". La "estructuración" no consiste más que en "la articulación de relaciones sociales por un tiempo y un espacio, en virtud de la dualidad de la estructura". (GIDDENS,1995a, p. 396)

b) El concepto de "acción" como "acción reflexiva", que Giddens toma de Garfinkel: "Los agentes humanos o actores tienen, como un aspecto intrínseco a lo que hacen, la aptitud de comprender lo que hacen en tanto lo hacen" (GIDDENS, 1995a, p. 396). Pero la reflexividad sólo en parte opera en un nivel discursivo; muchas veces los actores saben qué hacer y qué no hacer en determinados contextos sociales, sin ser capaces por ello, de asociar a esos comportamientos una expresión discursiva. Esto lleva a la distinción entre "conciencia discursiva" y "conciencia práctica", donde la segunda tiene una importancia central: más que lo que los individuos dicen hacer, importa lo que en realidad hacen, por lo cual la teoría enfatiza más la "acción" que al "actor".

Pero como muestra Margaret Archer (2003) la relación entre acción y estructura, no queda saldada a través de la teoría de la estructuración. ¿Por qué frente a las mismas constricciones y propiedades habilitantes, unos sujetos emprenden unos cursos de acción y otros, otro muy distinto? Su idea de una "conversación interna", que mantienen los sujetos consigo mismos, ilumina el modo como los agentes son influidos por la estructura y a la vez actúan sobre esta, modificándola. Esta puesta en primer plano de los procesos deliberativos internos de sujetos más o menos reflexivos, es crucial para comprender las razones por las que, personas concretas, se posicionan de modos peculiares respecto de sus posibilidades dentro de la estructura, sin utilizarlas tanto como 
podrían, contrariándolas, o haciendo esfuerzos sobrehumanos para sobreponerse a los aspectos restrictivos de la misma, a fin de definir un proyecto personal propio y llevarlo a cabo.

Los factores sociales y culturales no actúan de por sí, sino que deben su influencia a las propiedades y poderes de los agentes, propiedades y poderes - pensar, deliberar, querer - que sólo son característicos y distintivos de las personas. Para Archer, las constricciones y los recursos (fuerzas causales de propiedades estructurales emergentes, tales como los roles, las organizaciones y las instituciones, o de propiedades culturales emergentes, tales como proposiciones, teorías o doctrinas) no pueden actuar por sí mismos, como si fueran "entidades". Para que algo pueda actuar como constricción o como recurso, debe ser puesto en una relación tal que obstruya o ayude al logro de alguna actividad específica del agente. Como sólo las personas poseen la intencionalidad de definir y diseñar cursos de acción para lograr sus propios objetivos, no es posible referirse a las constricciones y los recursos sino es en relación con agentes que tienen proyectos, y los persiguen. Desde este punto de vista, entonces, es crucial distinguir entre la existencia de propiedades estructurales y el ejercicio de sus fuerzas causales.

En suma, para que los factores estructurales y culturales puedan ejercer su poder constreñidor o recursivo, se requiere de: a) la existencia de proyectos humanos; b) una relación de congruencia o incongruencia con los proyectos particulares de los agentes; c) respuestas de los agentes a estas influencias, las que, al ser condicionantes más que determinísticas, están sujetas a la deliberación reflexiva sobre la naturaleza de la respuesta. (ARCHER, 2003, p. 8). El mecanismo mediador entre estructura y agencia, y que permite explicar el condicionamiento social, es la "conversación interna" por la cual nos es posible definir modos de vida en condiciones sociales objetivas que no podemos elegir.

Estos procesos reflexivos de los agentes tienen expresiones prácticas y discursivas, que tanto en el caso de los alumnos como de los docentes, asumen a veces la forma de posicionamientos antagónicos recíprocos.

\section{Estructura, acción y sujeto en el espacio escolar: la erosión de la normativa escolar y el múltiple afloramiento de lo social}

Las normas, bien es sabido, se confirman en su vigencia por su cumplimiento. El desafío de esas normas y de los límites de la acción permitida por parte de los adolescentes, es un modo de conocer al mundo social en el que se mueven y de probar sus propias capacidades. La Psicología ha abundado en la importancia de los límites en la formación de la identidad durante la infancia y la juventud, pero también la Sociología ha generado teoría e investigación sobre los beneficios psicológicos de las limitaciones que emanan de la vigencia de un cuerpo de normas que estructura el espacio social y le da sentido a la acción. Ya repasamos las concepciones de Giddens y Archer al respecto. Antes que 


\section{Adriana Marrero}

ellos, Durkheim (1947), señala el modo cómo la limitación de los deseos permite satisfacerlos, y cómo los límites normativos proveen de la capacidad de contener y brindar seguridad a unos sujetos que, en la modernidad, se ven amenazados por la anomia:

El conjunto de reglas [...] forma verdaderamente alrededor de cada hombre una especie de barrera ideal, al pie de la cual vienen a morir el torrente de las pasiones humanas, sin poder avanzar más allá. Y sólo es posible llegar a satisfacerlas porque están contenidas. (DURKHEIM, 1947, p. 48)

Y en concordancia con la actual idea de estructura, sostiene que "Lejos de excluirse regla y libertad como términos antinómicos, el segundo sólo es posible con el primero" (DURKHEIM, 1947, p. 59).

En el campo educativo, esto significa, sin más, que no puede hablarse de libertad de los estudiantes y de los docentes (y de su capacidad de actuar), sin referirnos simultáneamente a la vigencia de un sistema normativo que estructure el espacio social de la escuela y que les brinde las certezas y los recursos para orientarse en las múltiples relaciones sociales que deben entablar en la vida liceal, mediante sus propios procesos reflexivos internos.

Por otro lado, es importante subrayar que es la vigencia real de las normas lo que genera el campo para la trasgresión, y es la pérdida de su vigencia, lo que lo liquida. Cuando están vigentes, las reglas brindan, sobre todo, certezas que limitan la posibilidad de elección. Por ejemplo, la obligación de asistir a la escuela cotidianamente, no sólo elimina del horizonte de posibilidad cotidiano, la idea de no concurrir; también convierte en trasgresión la no asistencia sin razón justificada. La "rabona", "la rata" o como quiera llamarse a las inasistencias individuales o grupales voluntarias, con fines de ocio, sólo es posible como trasgresión, si la obligación de asistir está vigente y es firme. Pero si es azarosa, intermitente, o inconsecuente, - del lado de los estudiantes o de los docentes la "rabona" pierde visibilidad y hasta existencia: ¿Qué "rabona" es la que tiene lugar cuando no hay clases porque el docente no fue? ¿O cuando no se pasa lista? Hay otras muchas trasgresiones escolares que dejan de existir cuando se afloja la normativa escolar: el incumplimiento con la tarea, sólo es trasgresor, cuando hay tareas y los docentes las corrigen y las comenten. Si no hay trabajos encargados, el no trabajar no es trasgredir. En un contexto institucional de lasitud normativa, no hay trasgresión posible. Al menos no, dentro del campo específico de lo escolar.

Ahora bien, es sabido que la trasgresión, sobre todo en la adolescencia, es un comportamiento de puesta a prueba de la vigencia de las normas, del testeo de los límites dentro de los cuales es seguro el comportamiento, lo que contiene la conducta y facilita la constitución de la identidad juvenil. ¿Puede la 
Pluralismo, estructuración y construcción de la identidad en la educación media uruguaya: interacciones desde las trincheras

debilidad estructural del espacio educativo clausurar los espacios de trasgresión? ¿Cómo se procesan estas trasgresiones si desaparecen los campos donde las mismas pueden ser ejercitadas?

La erosión de la normativa escolar - de las formas específicas de estructuración del espacio educativo - está lejos de significar una erosión de toda normativa; lejos de ello, la erosión de las normas escolares deja en evidencia la vigencia de formas estructurantes ajenas a la escuela, de carácter social, y la pluralidad de significados que es característica de las sociedades actuales, tal como las describimos más arriba. En otras palabras, el retiro de la escuela de su pretensión - y su práctica - estructurante, permite la manifestación y emergencia de lógicas de comportamiento múltiples, frecuentemente contrapuestas, relativas a contextos sociales y culturales diferentes, que no sólo pertenecen a los distintos tipos de estudiantes, sino también a los distintos tipos de docentes y de adultos que se encuentran, y a veces chocan, dentro de la escuela.

La red de reglas recursivas formalizadas y previsibles de la organización escolar moderna, sin desaparecer, se interrumpe allí donde se debilita, porque le cortan el paso otros sistemas de reglas - más o menos vigorosos y también fragmentados - que viven dentro de la escuela incorporados por distintos tipos de alumnos y de docentes, igual que fuera de ella. Sin la fuerza vinculante de la legitimidad de la regla escolar, cada una de estas intersecciones se convierte en un punto de lucha y un foco de tensión - entre docentes y alumnos, alumnos entre sí, docentes y padres, etc. - por la definición de qué es lo normativamente válido y exigible y qué no lo es. Contrariamente a lo que se argumenta algunas veces, estas lógicas de estructuración del comportamiento no irrumpen súbitamente en el espacio escolar, - cuando se incendia una papelera, se eructa en clase, o se llama a la policía - sino que le pre-existen. Por eso hablamos de afloramiento. Son formas de estructuración del espacio social sobre el cual se construye la institución educativa, procurando instaurar otras nuevas, más pacíficas, más civilizadas, más complejas. Es la erosión de esta última - o su fracaso en constituirse como la principal fuerza estructurante del comportamiento dentro de ese espacio dado - la que deja ver, como a las rocas que asoman bajo la arena, estas formas persistentes de estructuración social.

La prevalencia de una cultura masculina apoyada en la fuerza física de la que también participan algunas mujeres, el anti-intelectualismo que diagnosticaba ya Willis en "Aprendiendo a trabajar" (WILLIS, 1988), el pillaje y el vandalismo como modo de apropiación o agravio a un mundo que se siente extraño, el consumo de alcohol y de drogas en los espacios educativos, son algunas de las evidencias de una de las varias formas culturales coexistentes en la escuela. Recordemos el curioso escenario en el que se desarrollaba el examen de didáctica descrito por una docente en las primeras páginas de este artículo. Ese solo caso expresa, mejor que cualquier otro, este afloramiento de pautas que no son específicas del espacio escolar, pero que lo han colonizado, 
al parecer completamente, desde las paredes garabateadas, hasta el comportamiento manifiestamente indecoroso de los estudiantes; $y$ esto, al parecer, sin resistencia alguna de los actores adultos, educadores, expertos en didáctica.

Pero también hay otras pautas - múltiples y contradictorias - que afloran con el retiro de los modos de estructuración específicamente escolares, como lo es, por ejemplo, la cultura del relativismo nihilista y permisivo de algunos docentes, que culmina en una suerte de reproductivismo tácito, por el cual estos se niegan a sí mismos el derecho de educar a nadie. Como decía, hace años, un profesor de tecnología: "Si viven de criar chanchos... ¿ cómo voy a enseñarles tecnología?". O, como lo ilustraba el primero de los casos presentados, la cultura represiva y punitiva que usa el recurso a la fuerza policial como único mecanismo disponible ante el olvido de la función educativa de la escuela y de sus propios métodos para la formación de los adolescentes. Tal vez sea éste, el caso en el que más claramente se manifieste la irrupción dentro del espacio educativo de lógicas que no son educativas: los estudiantes trasgresores no son ni educados, ni castigados porque las clases, simplemente, se suspenden; los buenos estudiantes se ven castigados primero y privados de clases después; los docentes, directores y autoridades de la enseñanza, no se consideran responsables de nada y reclaman que no se les descuenten estos dos días extras "de vacaciones". Tampoco era educativa la pauta de relacionamiento que imponía aquella ex profesora de práctica, en el solapado conflicto entablado con los estudiantes, con las autoridades, y tal vez consigo misma, por su derecho a no impartir unos contenidos que terminaba no impartiendo, sostenido por una peculiar alianza con los malos alumnos.

\section{En suma...}

Posicionados como sujetos diestros en la interpretación de un mundo escolar que algunas veces no parece tener sentido alguno, los jóvenes entablan, como pueden, $y$ desde donde pueden, en las propias trincheras de sus particulares posiciones sociales y mundos de significación, sus luchas personales por un espacio y por un tiempo que reclaman como propio: ya sea para estudiar y entonces, desde la propia y declinante normativa escolar, reclaman a los docentes asiduidad, seriedad y respeto y a sus compañeros, silencio y colaboración, y al sistema, que funcione de acuerdo con lo establecido. O para luchar contra el anonimato, la alienación y el olvido, y entonces colonizan los espacios comunes - muros, bancos, puertas - resignificándolos como lienzos, para el despliegue creativo, como pancartas para la reivindicación, el exabrupto, o el insulto; o como simples rincones donde dejar grabados poemas, corazones, o iniciales, esperando que esos mensajes lleguen a alguna parte. O para ganar ocio, y entonces, entablan contra el docente, una lucha a veces velada y otras manifiesta, por el uso del tiempo y la duración de la clase, por el tema de conversación, por el dominio de la situación, generando problemas, o planteándolos, creando distracciones, o aliándose, como vimos, con docentes 
Pluralismo, estructuración y construcción de la identidad en la educación media uruguaya: interacciones desde las trincheras

afines a sus luchas. O por la elemental necesidad de construcción identitaria en la adolescencia, que se conforma también en un juego antagónico donde se prueban las propias fuerzas, y entonces se desafía cualquier regla que esté a la vista. Como regla recursiva característica de los múltiples procesos de estructuración que se procesan desde fuera y dentro del espacio escolar, cada una de las trincheras se convierte en lugar de espera y defensa, de protección y eventual ataque, de tránsito y de permanencia, pero siempre de proveedor de certezas en el cruce de pautas diversas y caóticas.

Pero estas no son conductas irreflexivas, ni, como muchas veces se dice - cuando es el caso - formas inéditas de violencia o agresión. Desde sus propios lugares, los jóvenes se preguntan, se responden, actúan, reflexionan a veces con mejor o peor suerte sobre sus situaciones presentes y sus posibilidades futuras, sobre su capacidad para aprovechar sus oportunidades, o para desafiar y contrariar las constricciones de las que son sólo parcialmente conscientes. Muchas veces, las formas de agresión y violencia que son visibles en los espacios escolares y en su periferia, así como otras manifestaciones menos notorias pero igualmente negadoras del espacio escolar, como es el abandono, no son sino resultado de respuestas propias, no menos lúcidas, a las preguntas que estos mismos jóvenes se hacen sobre su situación actual y a las intuiciones que pueden formarse sobre su futuro. Mientras tanto, luchan, atacan, resisten, o simplemente se defienden, en las trincheras del complejo y fracturado entramado normativo de un espacio escolar en retirada.

\section{Referências}

ARCHER, M. Structure, Agency and the Internal Conversation. Cambridge: Cambridge University Press, 2003.

BERGER, P. El dosel sagrado. Elementos para una sociología de la religión. Buenos Aires: Amorrortu, 1971.

BERGER, P.; Y LUCKMANN, T. Modernidad, pluralismo y crisis de sentido. Barcelona: Paidós, 1997.

DURKHEIM, E. La educación moral. Buenos Aires: Paidós, 1947.

GIDDENS, A. Consecuencias de la modernidad. Madrid: Alianza, 1994. . La constitución de la sociedad. Buenos Aires: Amorrortu, 1995a. Modernidad e Identidad del yo. Barcelona: Península, 1995b.

LIPOVETSKY, G. EI crepúsculo del deber. Barcelona: Anagrama, 1994.

La era del vacío. Barcelona: Anagrama, 1986.

MARRERO, A. Educación y modernidad, hoy. Montevideo: Germanía-EBO, 2007. 


\section{Adriana Marrero}

MARRERO, A. El bachillerato uruguayo. Montevideo: CSIC-UdelaR, 2008.

RIESMAN, D. La muchedumbre solitaria. Buenos Aires: Paidós, 1964.

WILLIS, P. Aprendiendo a trabajar. Madrid: Akal, 1988.

Los soldados rasos de la modernidad. La dialéctica del consumo cultural y la escuela del siglo XXI. en Marrero, A. 2007.

\section{Correspondência}

Adriana Marrero - Depto. de Sociología, Facultad de Ciencias Sociales, Universidad de la República, Constituyente 1502 Of. 515, 11200 Montevideo.

E-mail: adriana.marrero@gmail.com

Recebido em 15 de novembro de 2010

Aprovado em 11 de fevereiro de 2011 